\title{
Thérapie génique du déficit immunitaire combiné sévère lié à $L X$
}

\author{
A. Fischer \\ Unité d'Immunologie et d'Hématologie Pédiatriques, Département de Pédiatrie, Hôpital Necker, 149, rue de Sèvres, F- \\ 75743 Paris cedex 15, France
}

Les déficits immunitaires combinés sévères sont les formes les plus sévères de déficits immunitaires héréditaires. Ils se caractérisent par l'absence de différenciation des lymphocytes $T$, cellules indispensables à la défense contre la plupart des micro-organismes. Plusieurs de ces maladies sont aujourd'hui caractérisées sur le plan génétique et moléculaire. Il en est par exemple ainsi du déficit en adénosine désaminase et du déficit immunitaire combiné sévère lié au chromosome $X$. Cette dernière forme de déficit immunitaire combiné sévère (DICS) est la plus fréquente. Le gène codant pour la chaîne $\ddot{Y}_{c}$ des récepteurs des cytokines interleukine 2, 4, 7, 9 et 15 est muté dans cette maladie. Le déficit d'interaction entre les cytokines IL7 et IL15 avec leur récepteur respectif sont responsables des défauts de production de lymphocytes $T$ et de lymphocytes NK dans cette maladie.

Le traitement actuel de cette maladie repose sur l'allogreffe de moelle osseuse. Lorsqu'un donneur HLA identique peut être identifié dans la famille (environ une fois sur cinq), la probabilité de succès de cette thérapeutique est voisine de $100 \%$. Dans les autres cas, sont $80 \%$ des malades, la probabilité de succès d'une greffe de moelle osseuse provenant d'un donneur compatible non apparenté, ou d'un donneur partiellement incompatible dans la famille, est de l'ordre de 50 à $60 \%$. Les échecs sont liés aux infections et à la réaction du greffon contre l'hôte.

Il est de ce fait justifié de rechercher à mettre au point une autre forme de traitement de cette maladie, il s'agit du traitement par transfert de gène dans les cellules souches hématopoïétiques. Le principe consiste à infecter les cellules hématopoïétiques médullaires de ces patients par un vecteur rétroviral contenant le gène codant pour la chaîne $\ddot{Y} c$. II est escompté que l'infection par le rétrovirus permette l'intégration du provirus contenant le gène $\ddot{Y} c$ dans le génome de la cellule et son expression dans les cellules filles.

A ce jour, nous avons obtenu une série de résultats encourageants dans cette perspective : nous avons montré qu'à l'aide d'un vecteur rétroviral défectif, il était possible d'obtenir l'expression stable de la chaîne Ÿc dans des lymphocytes $B$ de patients transformés par le virus d'Epstein Barr. La chaîne Ÿc est exprimée à la surface de la cellule et est fonctionnelle, le niveau d'expression est satisfaisant, et cette expression est stable pendant plusieurs mois.

De même il a été possible à l'aide du même vecteur rétroviral d'obtenir l'expression de la chaîne Ÿc dans des progéniteurs hématopoïétiques donnant naissance à des lymphocytes Natural Killer.

En parallèle, nous avons entrepris des expériences dans un modèle murin de la maladie qui a été généré par la technique d'inactivation génique par recombinaison homologue. Le transfert du gène $\ddot{Y} c$ dans les cellules hématopoïétiques médullaires de cette souris puis la réinjection in vivo permet, selon des résultats préliminaires, la correction de la maladie.

Le projet de recherche actuel cherche dans sa phase pré-clinique a d'une part optimiser les conditions de transfert de gène in vitro dans les cellules des patients, d'autre part déterminer dans le modèle animal murin s'il est possible d'observer un avantage sélectif des cellules exprimant le gène Ÿc, dites "cellules transduites". La première partie du projet consiste à tester plusieurs paramètres de l'infeciton des cellules par la source de vecteur rétroviral afin d'augmenter le nombre de cellules infectées, sont testés : l'intérêt d'une cocentrifugation des cellules avec le surnageant viral ; l'utilisation de cellules stromales autologues ; différentes températures ; différents nombres de cycles d'infection ; l'utilisation de milieux sans sérum ; l'addition de fibronectine.

Ces différentes expériences devraient permettre dans l'année à venir de déterminer le meilleur protocole possible en vue d'une application clinique.

La deuxième partie du projet de recherche consiste à répéter les expériences de transfert de gène in vivo 
chez la souris déficiente en $\ddot{Y} c$ dans des conditions où le nombre des cellules infectées est faible. Ces expériences sont déterminantes afin de savoir si un taux de transfert de gène faible peut donner naissance néanmoins à un nombre suffisant de lymphocytes $T$ corrigeant la maladie. Cette expérience est cruciale pour l'application humaine car nous savons que le transfert de gène dans les cellules hématopoïétiques humaines, contrairement à la souris est relativement peut efficace. La démonstration d'une avantage sélectif des cellules transduites dans le modèle murin, en l'absence de toute toxicité permettrait d'envisager un premier essai clinique chez l'homme.

Hematol Cell Ther (1997) 39: 97

(c) Springer-Verlag France 1997 